Research Article

\title{
Accurate Non-rigid Registration of Lung Images Based on Mutual Information
}

\author{
Shangli Cheng, Daxiang Cui \\ Institute of Nano Biomedicine and Engineering, Department of Instrument Science and Engineering, School of Electronic \\ Information and Electrical Engineering, Shanghai Jiaotong University, Shanghai 200240, China \\ Corresponding author: E-mail: dxcui@sjtu.edu.cn
}

Received: Nov. II, 20I5; Accepted: Dec. 7, 2015; Published: Dec. 14, 2015.

Citation: Shangli Cheng and Daxiang Cui. Accurate Non-rigid Registration of Lung Images Based on Mutual Information. Nano Biomed. Eng. 2015, 7(4), 153-159.

DOI: 10.5101/nbe.v7i4.pI53-159.

\begin{abstract}
In clinical diagnosis of lung disease, registration of lung images from different imaging systems can provide a multi-informative image and improve its diagnostic accuracy. In this study, the original lung images were obtained from computed tomography (CT) and single-photon emission computed tomography (SPECT). After the decomposition of the images using wavelet transform, a non-rigid registration were proposed, in which CT image was used as the reference image and SPECT image was used as the floating image. In registration, the mutual information between the reference and floating images was calculated in the process of translation, rotation and elastic transformation. At last, the result of the registration was evaluated by the edges of the bone, muscle, thorax and lung tissues in CT and SPECT images. It showed an accuracy registration between lung CT and SPECT images.
\end{abstract}

Keywords: Lung disease; CT; SPECT; Non-rigid registration; Mutual information

\section{Introduction}

The registration of the images is to overlay the images which were obtained from different imaging systems, at different time and by different sensors $[1,2]$. Especially in clinical medicine, the image registration, such as computed tomography (CT), single-photon emission computed tomography (SPECT) and positron emission computed tomography (PET), is a basic tool for imaging applications in diagnosis [3]. In diagnosis of lung disease, registration of lung images is increasingly used $[4,5]$. For example, registration of the CT images of the same patient, which were scanned at different time, was used to evaluate the development of the lung disease $[6,7]$, and registration of the CT,
SPECT and dose map images was used for radiation therapy $[8,9]$.

The lung CT image contains the anatomic information. Comparatively, the radioactive focus, sensitively reflecting the lung function, can be detected using SPECT images [10]. Registration of the CT and SPECT images would provide the multi-information in diagnosis or therapy of lung disease. The application of registration in lung disease would lead great helps in diagnosis and therapy of lung disease. For registration of the images, the main steps are the image transformation and the image matching [1]. Many methods of image transformation had been developed in registration of lung images, including rigid and non- 
rigid methods [11-13]. However, less researches were carried on in the registration of lung CT and SPECT images $[14,15]$.

In this study, we proposed an accuracy elastic registration method for lung CT and SPECT images based on mutual information. The registration result was further evaluated by the edges of the bone, muscle, thorax and lung.

\section{Materials and Methods}

\section{The image data and preprocessing}

The original images were obtained from computed tomography (CT) and single-photon emission computed tomography (SPECT). The images of CT and SPECT were from the same volunteer at chest. The gray level of the images was 256 , which was from 0 to 255 . The pixels of lung CT image was $720 \times 677$, and the pixels of lung SPECT image was $1080 \times 1015$. As the original images had redundant pixels of black background, the images were segmented. At last, the CT images with pixels of $571 \times 571$ and the SPECT images with pixels of $571 \times 571$ were used. In CT image, the bones and the muscles were imaged clearly, and the lung was not imaged. In SPECT image, the lung was imaged clearly. In this case, the registration of the two images could help in the diagnosis of lung disease.

\section{The mutual information of two gray images}

In the registration of two images, the mutual information (MI) was used to evaluate the registration accuracy of the two images $[16,17]$. For the gray images of $\mathrm{A}$ and $\mathrm{B}$, the mutual information $\mathrm{MI}(\mathrm{A}, \mathrm{B})$ was defined as

$$
\mathrm{MI}(\mathrm{A}, \mathrm{B})=\mathrm{H}(\mathrm{A})+\mathrm{H}(\mathrm{B})-\mathrm{H}(\mathrm{A}, \mathrm{B})
$$

The $\mathrm{H}(\mathrm{A})$ and $\mathrm{H}(\mathrm{B})$ was the information entropy of image $\mathrm{A}$ and $\mathrm{B}$, and $\mathrm{H}(\mathrm{A}, \mathrm{B})$ was the joint information entropy of the image $A$ and $B$. They were defined as:

$$
\begin{gathered}
\mathrm{H}(\mathrm{A})=-\sum_{a \in A} p_{A}(a) \log p_{A}(a) \\
\mathrm{H}(\mathrm{B})=-\sum_{b \in B} p_{B}(b) \log p_{B}(b) \\
\mathrm{H}(\mathrm{A}, \mathrm{B})=-\sum_{a \in A b \in B} p_{A B}(a, b) \log p_{A B}(a, b)
\end{gathered}
$$

where, $p_{A}(a)$ and $p_{B}(b)$ represent the gray probability distribution. The gray probability distribution was calculated based on the gray scale histogram, which is the distribution of the pixels in the image over the gray-level scale. The color intensity was from 0 to 255 . For image A, the gray probability of color intensity of a was defined as $p_{A}(a) \cdot p_{A B}(a, b)$ is the joint probability distribution of the two images. And the gray probability distribution $p_{A B}(a, b)$ was described by:

$$
p_{A B}(a, b)=\frac{h(a, b)}{\sum_{a, b} h(a, b)}
$$

where, $h(a, b)$ is the joint gray scale histogram of image $\mathrm{A}$ and $\mathrm{B}$.

In the process of registration of two images, the larger MI of the two images is obtained, the higher accuracy of the registration is. In this study, in order to reduce the error caused by the images from different imaging systems, the normalized MI was used and defined as:

$$
\operatorname{MI}(\mathrm{A}, \mathrm{B})=(\mathrm{H}(\mathrm{A})+\mathrm{H}(\mathrm{B})) / \mathrm{H}(\mathrm{A}, \mathrm{B})
$$

\section{The decomposition of the image based on wavelet transform}

The wavelet transform was the time-frequency transformation. The wavelet analyses were wildly used in image registration, which could speed up the calculation [18]. The 2-dimensional discrete wavelet transform (DWT) was suit for the CT and SPECT images $[19,20]$. By the wavelet transform, the image was decomposed into the high-frequency portions and the low-frequency portion:

$$
\begin{aligned}
& \Phi_{L L}(x, y)=\Phi(x) \cdot \Phi(y) \\
& \Psi_{L H}(x, y)=\Phi(x) \cdot \Psi(y) \\
& \Psi_{H L}(x, y)=\Psi(x) \cdot \Phi(y) \\
& \Psi_{H H}(x, y)=\Psi(x) \cdot \Psi(y)
\end{aligned}
$$

where, the $\Phi_{L L}(x, y)$ was the low-frequency portion in the horizontal and vertical direction. $\Psi_{L H}(x, y)$ and $\Psi_{H L}(x, y)$ were the high-frequency portions in the horizontal and in the vertical direction, respectively. $\Psi_{H H}(x, y)$ was the high-frequency portion in the horizontal and vertical direction. The $\Psi(x)$ was the wavelet basis function. The $\Phi(x)$ was the scaling function. For a 2-dimensional image, the MALLAT fast transform algorithm can be used. The 2-dimensional image $f(x, y) \in V_{j}^{2}$ can be described as:

$$
f(x, y)=A_{j+1} f+D_{j+1}^{1} f+D_{j+1}^{2} f+D_{j+1}^{3} f
$$


where,

$$
\begin{gathered}
A_{j+1} f=\sum_{m_{1}, m_{2} \in Z} c_{j+1, m_{1}, m_{2}} \Phi_{j+1, m_{1}, m_{2}} \\
D_{j+1}^{\varepsilon} f=\sum_{m_{1}, m_{2} \in Z} D_{j+1, m_{1}, m_{2}}^{\varepsilon} \Psi_{j+1, m_{1}, m_{2}}^{\varepsilon}, \quad \varepsilon=1,2,3
\end{gathered}
$$

The $V_{j}^{2}(j \in \mathrm{Z})$ was discrete resolution analysis of $L^{2}(R)$. The standard orthogonal set of $L^{2}(R)$ was constituted by the wavelet function set of $\left[\Psi_{\mathrm{m}_{1}}\right.$, $\left.{ }_{\mathrm{m}_{2}} \mid\left(\mathrm{m}_{1}, \mathrm{~m}_{2}\right) \in \mathrm{Z}^{2}\right]$. The standard orthogonal set of $V_{j}^{2}$ was constituted by $\left[\Phi_{\mathrm{j}, \mathrm{m}_{1}, \mathrm{~m}_{2}} \mid j \in \mathrm{Z},\left(\mathrm{m}_{1}, \mathrm{~m}_{2}\right) \in \mathrm{Z}^{2}\right]$. $\mathrm{Z}$ represents the positive integer set. Hence, the 2-dimensional MALLAT decompositions of the image was defined as:

$$
\begin{aligned}
c_{j+1} & =H_{r} H_{c} c_{j} \\
D_{j+1}^{1} & =H_{r} H_{c} c_{j} \\
D_{j+1}^{1} & =G_{r} H_{c} c_{j} \\
D_{j+1}^{3} & =G_{r} G_{c} c_{j}
\end{aligned}
$$

where $c_{j+1}, D^{1}{ }_{j+1}, D^{1}{ }_{j+1}$, and $D^{3}{ }_{j+1}$ represent the lowfrequency section, high-frequency in horizontal, highfrequency in vertical direction and high-frequency in diagonal direction. The $H_{r}$ and $G_{r}$ was set to express the filter operated on the row. The $H_{c}$ and $G_{c}$ to express the filter operated on the column. $H$ and $G$ was used to represent the low-pass and high-pass filtering, respectively.

\section{The model of the non-rigid registration}

For the medical images came from different imaging systems and different time, the position of the body would be changed, and the morphologic changes of the body were caused by the breathing. Under this circumstance, three method, including the translation of the image, the rotation of the image and the elastic registration of the image, were used in the non-rigid registration.

For the images of CT and SPECT, the CT image was used as the reference image (R) and the SPECT image was used as the corresponding floating image (F) because of more detail information of the tissues in the $\mathrm{CT}$ image. In the process of image registration, the floating image had the translation, rotation and elastic transformation to fit to the reference image. At the beginning, the translation of the floating image:

(1) Both the reference and floating images were decomposed using the wavelet transform. Then the high-frequency portions and the low-frequency portions of the reference and floating images would be obtained, named after R' and F', respectively.

(2) The $F^{\prime}$ was translated over the $R^{\prime}$ in the horizontal and vertical direction.

(3) All the portions of the $\mathrm{R}^{\prime}$ and $\mathrm{F}^{\prime}$ images were included. And the mutual information was calculated in the whole process of image translation.

(4) The F image was positioned in the new coordinate based on the maximum of the MI calculated by $R^{\prime}$ and $F^{\prime}$ images.

After the image translation, the floating image was rotated and the mutual information was calculated:

(1) The floating image with new coordinate was decomposed using the wavelet transform. Then the high-frequency portions and the low-frequency portions of the SPECT images would be obtained, named after F".

(2) The F" was rotated over the R' from 0 degree to 359 degree. The step was set to be 1 degree.

(3) The low- and high-frequency portions of the $\mathrm{R}^{\prime}$ and $\mathrm{F}^{\prime \prime}$ images were included. And the mutual information was calculated in the whole process of image rotation.

(4) The floating image was rotated in the new coordinate based on the maximum of the MI calculated by $\mathrm{R}^{\prime}$ and $\mathrm{F}^{\prime \prime}$ images.

The elastic transformation was carried on after the translation and rotation of the images.

(1) The floating image after the rotation process was decomposed using the wavelet transform. Then the high-frequency portions and the low-frequency portions of the floating images would be obtained, named after F"'.

(2) The elastic transformation of the F"' was in both horizontal and vertical directions.

(3) The mutual information was calculated in the process of elastic transformation. The ratio of the elastic transformation was less than 0.1 in order to avoid the distortion.

(4) The elastic transformation of the floating images was accepted when the MI between the R' and F"' images was extremum.

The process of the registration can be shown in Fig. 1. 


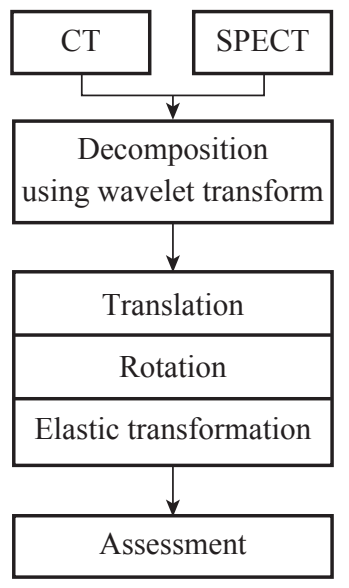

Fig. 1 Registration of lung CT and SPECT images.
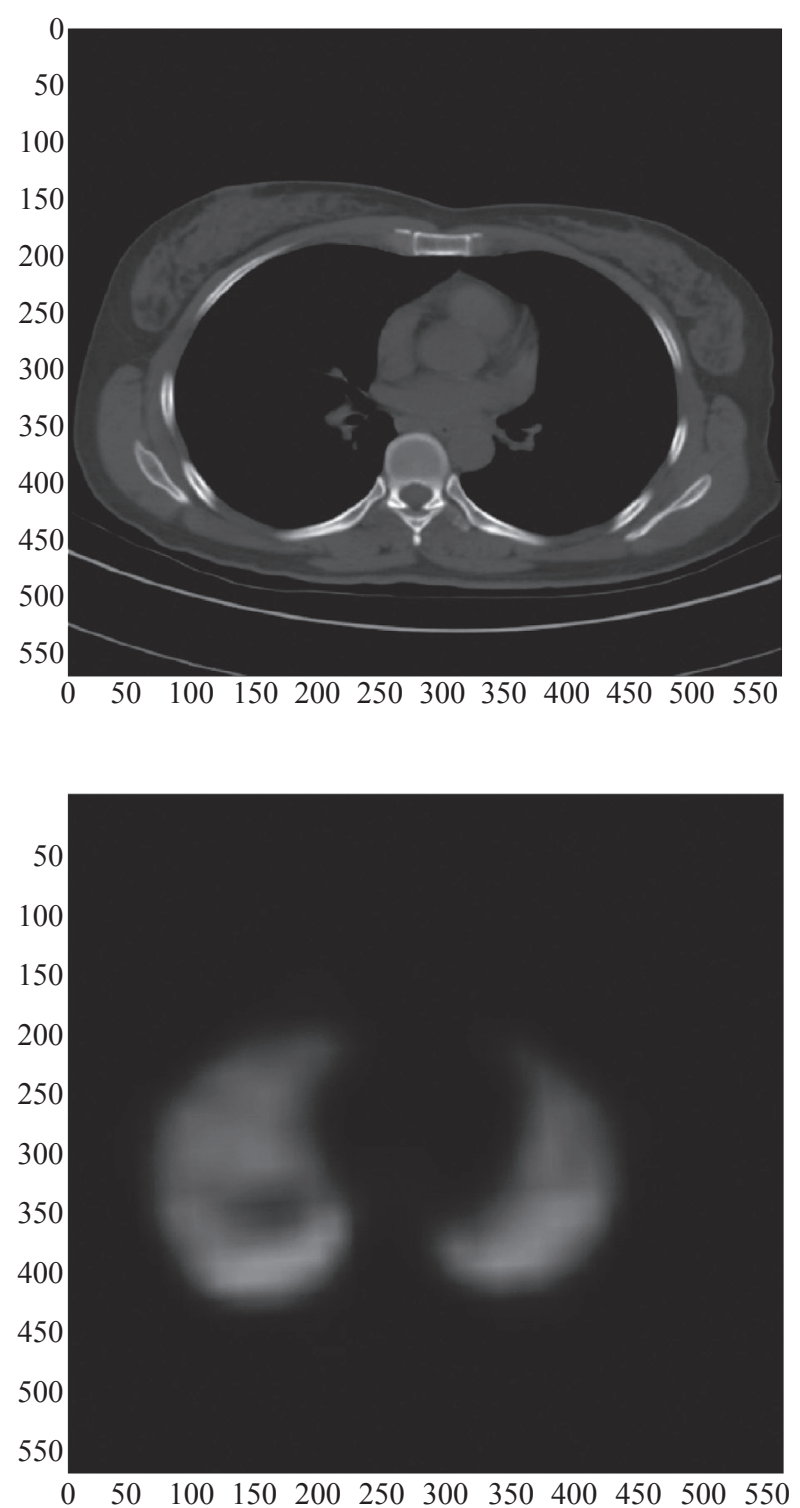

\section{The assessment of the registration}

The usual method to evaluate the registration of the images was visual assessment. As the medical images were from $\mathrm{CT}$ and SPECT imaging systems at chest. The discontinuity existed in the thorax, which was the main character in CT and SPECT images. As the edge is a set of points that have great difference in gray with the surrounding points, the edge of the thorax was extracted in both the CT and SPECT images after the process of registration. In the CT and SPECT images, a clear edge was between the bones, muscles and the thorax, it can be extracted and taken as the assessment
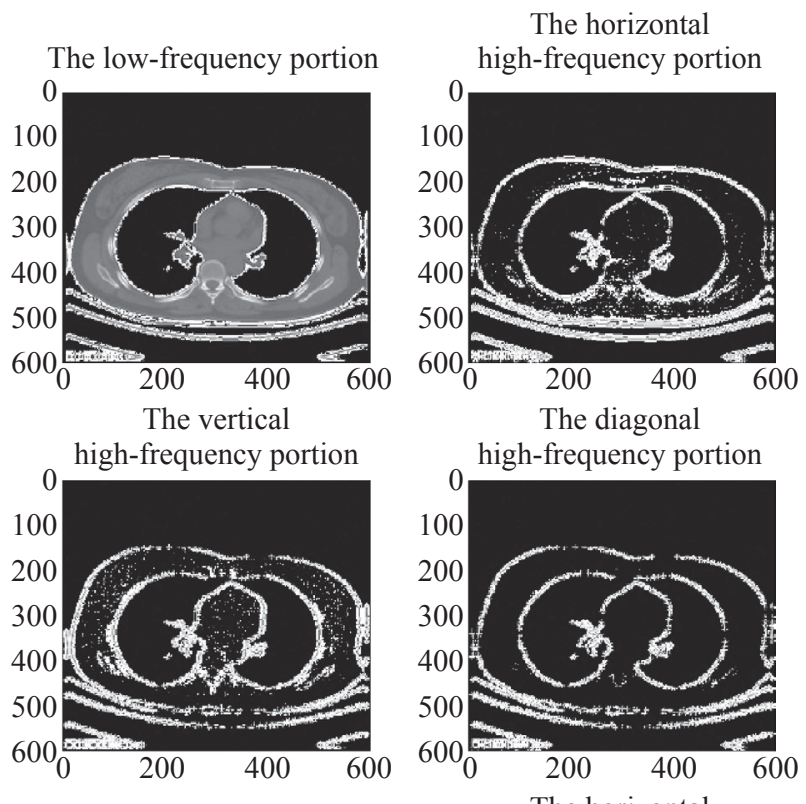

The low-frequency portion

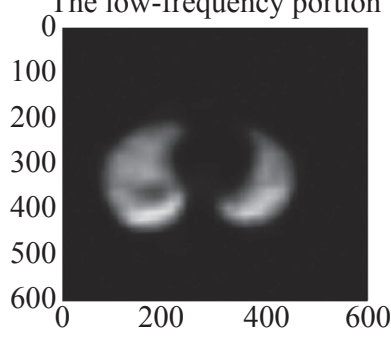

The veritical

high-frequency portion

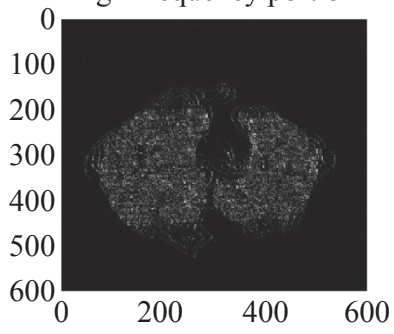

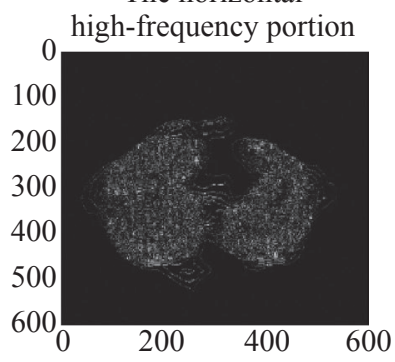

The diagonal high-frequency portion

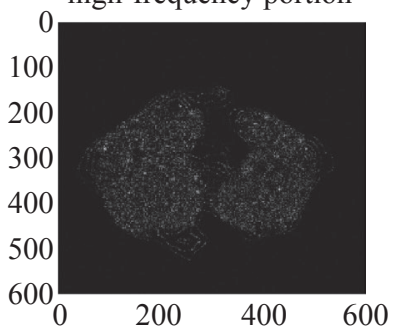

Fig. 2 The wavelet transform of the CT and SPECT images. (a) The original CT image. (b) The low-frequency portion of the CT image. (c) The horizontal high-frequency portion of the CT image. (d) The vertical high-frequency portion of the CT image. (e) The diagonal high-frequency portion of the CT image. (f) The original SPECT image. (g) The low-frequency portion of the SPECT image. (h) The horizontal high-frequency portion of the SPECT image. (i) The vertical high-frequency portion of the SPECT image. (j) The diagonal high-frequency portion of the SPECT image. 

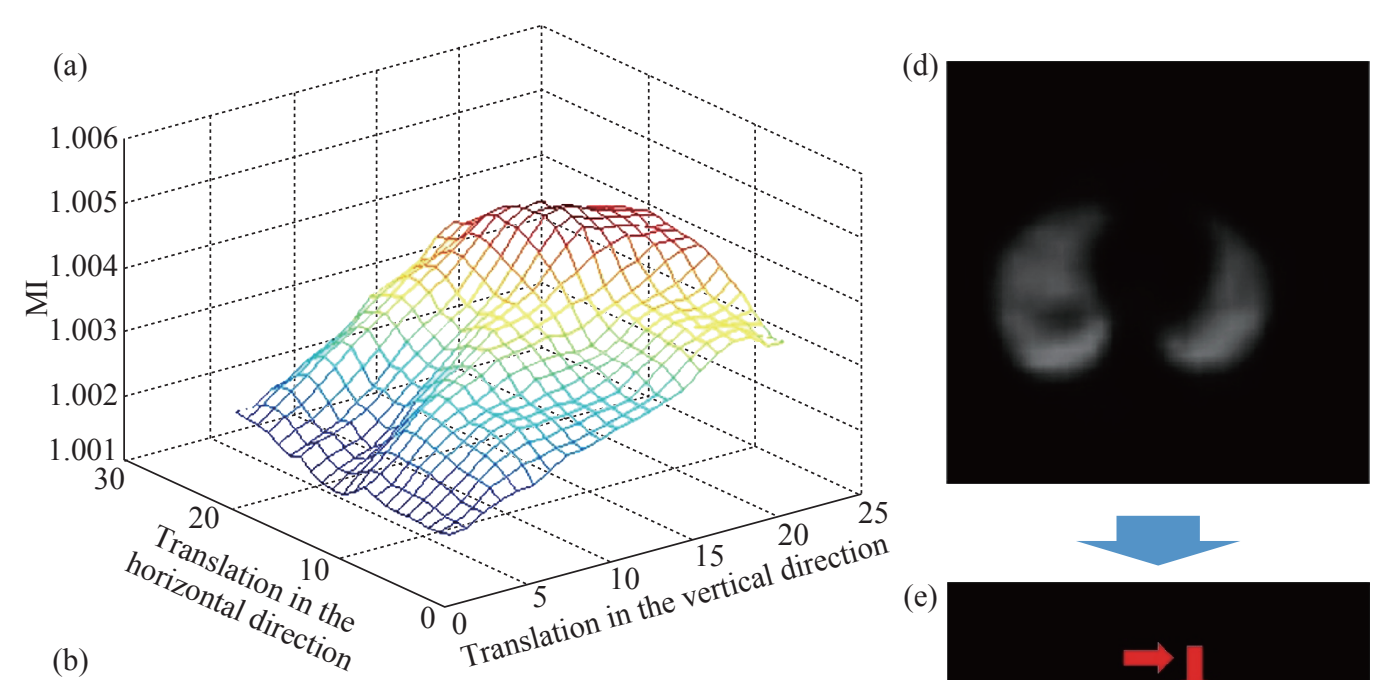

(e)
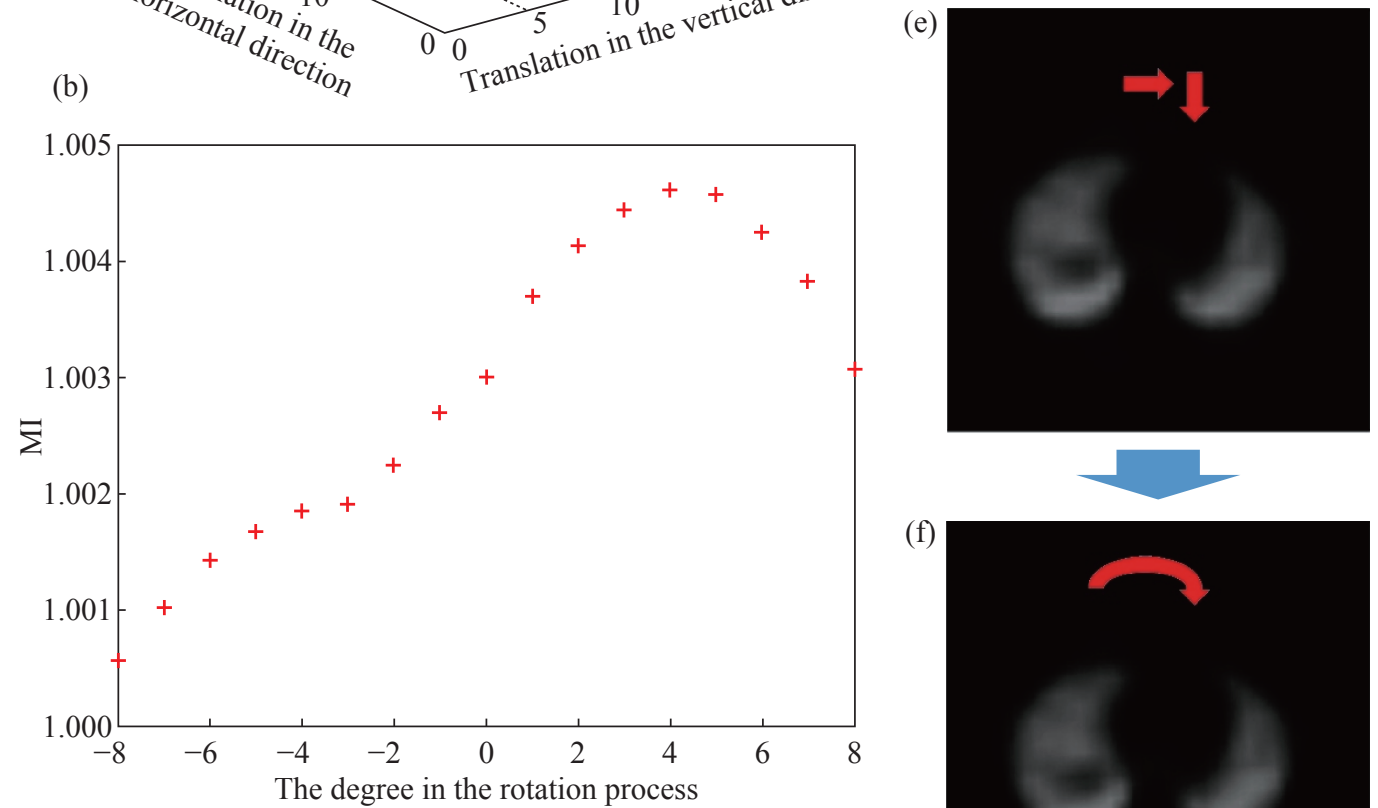

(f)
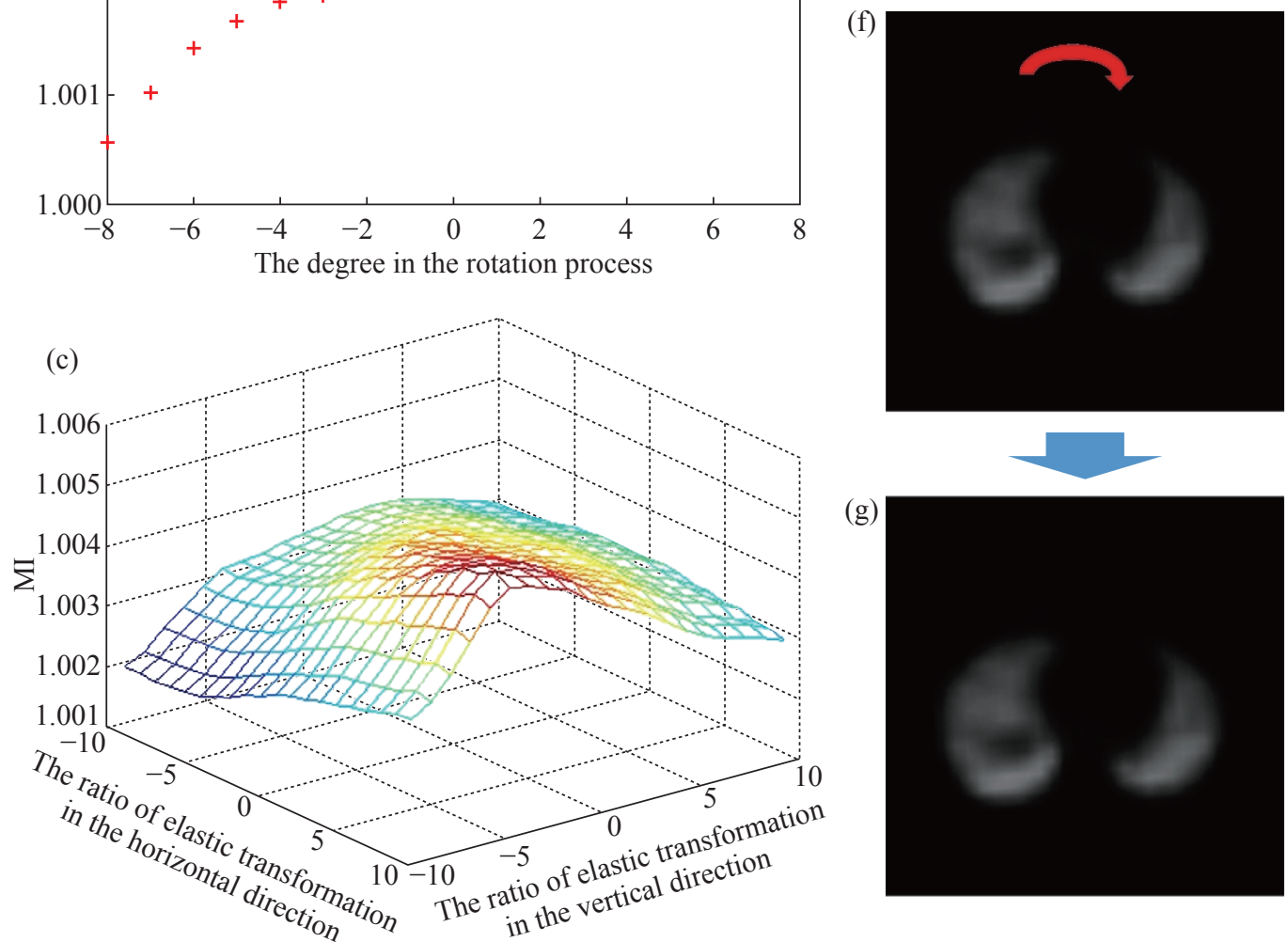

Fig. 3 The corresponding floating image. (a) The x-axis (unit: pixel) and y-axis (unit: pixel) represent the translation in the horizontal and vertical direction. The z-axis represents the mutual information. (b) The unit of the $\mathrm{x}$-axis is degree. The positive value means the rotation in clockwise, and vice verse. The y-axis represents the mutual information. (c) The x-axis (unit: percent, \%) and y-axis (unit: percent, \%) represent the elastic transformation ratio in the horizontal and vertical direction. The z-axis represents the mutual information. (d) The original SPECT image. (e) It shows the translation of the SPECT image based on the maximum of the MI. (f) It shows the rotation of the SPECT image based on the maximum of the MI. (g) It shows the elastic transformation of the SPECT image based on the maximum of the MI. The positive ratio meant the tension of the image, and the negative ratio meant the compression of the image. 
of the registration. The threshold of $\mathrm{T}$ was set, and the edge can be calculated by the binary image $\left(B_{A}(a)\right)$ :

$$
B_{A}(a)= \begin{cases}1 & A(a)>T \\ 0 & A(a)<T\end{cases}
$$

where, $A(a)$ is the gray value at the pixel of a. $\mathrm{T}$ is the threshold of gray value. The two colors used for a binary image are black and white. And ' 1 ' represents black, while ' 0 ' represents white. Based on the binary image, a clear edge between lung tissues and bones, muscles can be obtained. Then, the assessment would be based on the edges of the CT and SPECT images.

\section{Results and discussions}

The gray two images, including a CT image and a SPECT image at chest were used in the study. The resolution of the two images were $571 \times 571$. The gray level was 256 .

\section{The wavelet transform of the CT and SPECT images}

The CT and SPECT images were transformed into four portions, including the low-frequency portion, the horizontal high-frequency portion, the vertical highfrequency portion and the diagonal high-frequency portion (Fig. 2). The low-frequency portion was used in the non-rigid registration.

\section{The translation, rotation and elastic transformation of the SPECT image}

In order to acquire an accuracy image registration of CT and SPECT images, the floating step of the floating image was set to be one pixel and one degree in the process of translation and rotation, respectively. The CT image was used as the reference image, and the SPECT was the floating image (Fig. 3(d)). First, in the process of the translation of the SPECT image, the MI was calculated in the horizontal and vertical direction (Fig. 3(a)). The translation of the SPECT image was 13 pixel in the horizontal direction and 15 pixel in the vertical direction based on the maximum MI of 1.005 (Fig. 3(e)). Second, the SPECT image had the clockwise rotation of 4 degrees (Fig. 3(f)). Last, the SPECT was forced of horizontal tension and vertical compression by the maximum MI (Fig. 3(g)).

\section{The edges of the CT and SPECT images after the registration}

In order to evaluate the registration of the two images (Fig. 4(a)), the edge of the CT and SPECT were used. The edge of the thorax was extracted in the CT image, as well as the edge of the lung tissues in the SPECT image (Fig. 4(b)). The two edges was fitted greatly. It was proposed that the non-rigid registration, including the process of translation, rotation and elastic transformation, received an accuracy results in the CT and SPECT images at lung.

\section{Conclusions}

In this study, the non-rigid registration, including translation, rotation and elastic transformation, were well used in the medical images at lung. The method had little manual intervention and less amount of calculation. By the registration of the different medical (a)

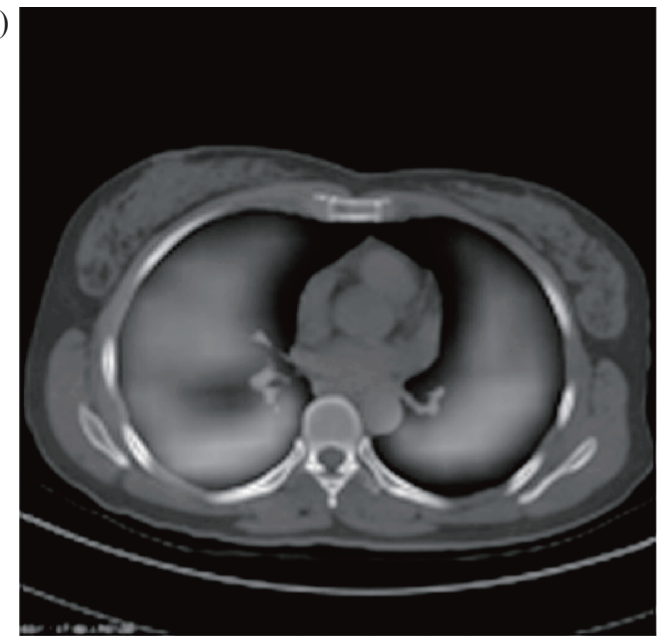

(b)

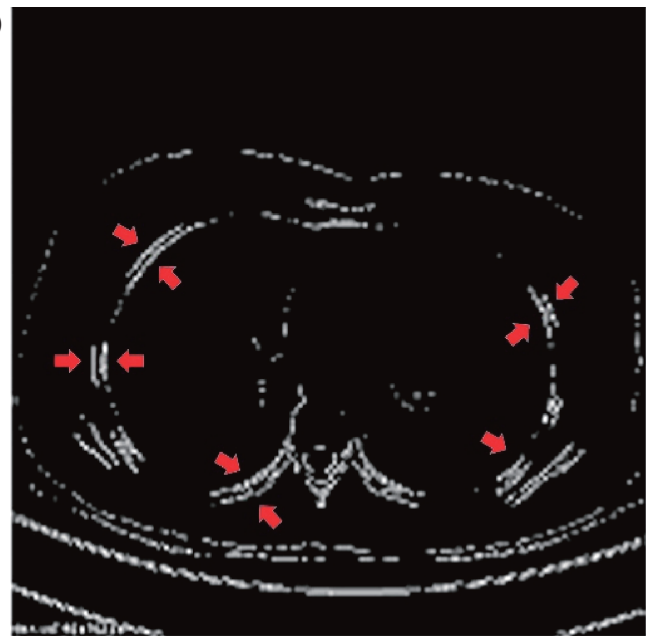

Fig. 4 The registration results and the edges of the CT and SPECT images after the registration. (a) The results of the registration. (b) The figure shows the edges of the CT and SPECT image after the registration. 
images at lung, the new image, consisting of bone, muscle and lung tissues, will bring great helps in the diagnosis of lung diseases.

\section{Acknowledgement}

This work is supported by National Key Basic Research Program (973 Project) (Project No. 20152015CB931802), National Natural Scientific Foundation of China (Grant Nos. 81225010, 81028009, and 31170961), 863 project of China (Project No. 2012AA022703 and 2014AA020700), and Shanghai Science and Technology Fund (13NM1401500).

\section{References}

[1] B. Zitova, J. Flusser, Image registration methods: a survey. Image Vision Comput, 2003, 21: 977-1000.

[2] F.P.M. Oliveira, J.M.R.S. Tavares, Medical image registration: a review. Comput Method Biomec, 2014, 17: 73-93.

[3] X.M. Cao, Q. Q. Ruan, A survey on evaluation methods for medical image registration. 2007 Ieee/ Icme International Conference on Complex Medical Engineering, 2007, 1: 718-721.

[4] L. Yin, L. Tang, G. Hamarneh, et al., Quantitative evaluation on the accuracy of image registration methods in SPECT guided radiation therapy for lung cancer patients. Med Phys, 2009, 36: 4312-4312.

[5] F. Courbon, O. Caselles, P. Giraud, et al., Application to lung conformal radiotherapy of a SPECT and computerised tomography (CT) image registration method. 13th International Conference on the Use of Computers in Radiation Therapy, 2000, 1: 141-142.

[6] Y.J. Zheng, K. Steiner, T. Bauer, et al., Lung nodule growth analysis from 3D CT data with a coupled segmentation and registration framework. Ieee I Conf Comp Vis, 2007, 1-6: 2511-2518.

[7] Z. Xue, K. Wong, S. T.C. Wong, Joint registration and segmentation of serial lung CT images for image-guided lung cancer diagnosis and therapy. Comput Med Imag Grap, 2010, 34: 55-60.

[8] H. Haneishi, N. Takita, D. Tsuchida, et al., Image registration between CT, SPECT and dose map images of lung and its application to image analysis in radiation therapy. 2003 IEEE Nuclear Science Symposium, 2004, 1: 2946-2950.

[9] N. Hardcastle, W. van Elmpt, D. De Ruysscher, et al., Accuracy of deformable image registration for contour propagation in adaptive lung radiotherapy. Radiation Oncology, 2013, 8: e243.

[10] L.S. Yin, L. Tang, G. Hamarneh, et al., Complexity and accuracy of image registration methods in SPECT-guided radiation therapy. Phys Med Biol, 2010, 55: 237-246.

[11] I. Sluimer, A. Schilham, M. Prokop, et al., Computer analysis of computed tomography scans of the lung: A survey. Ieee T Med Imaging, 2006, 25: 385-405.

[12] I. Sekine, H. Nokihara, N. Yamamoto, et al., Problems with registration-directed clinical trials for lung cancer in Japan. Tohoku Journal of Experimental Medicine, 2007, 213: 17-23.

[13] A. Al-Mayah, J. Moseley, M. Velec, et al., Deformable image registration of heterogeneous human lung incorporating the bronchial tree. Med Phys, 2010, 37: 4560-4571.

[14] L. Papp, M. Zuhayra, E. Henze, et al., Extended Normalized Mutual Information for Lung SPECT - CT Registration. 2009 3rd International Conference on Bioinformatics and Biomedical Engineering, 2009, 1-6: 1880-1882.

[15] F. Gutman, G. Hangard, I. Gardin, et al., Evaluation of a rigid registration method of lung perfusion SPECT and thoracic CT. American Journal of Roentgenology, 2005, 185: $1516-1524$.

[16] D. Tomazevic, B. Likar and F. Pernus, Multi-Feature Mutual Information Image Registration. Image Anal Stereol, 2012, 31: 43-53.

[17] M.M. Coselmon, J.M. Balter, D.L. McShan, et al., Mutual information based $\mathrm{CT}$ registration of the lung at exhale and inhale breathing states using thin-plate splines. Med Phys, 2004, 31: 2942-2948.

[18] J.M. Deng, H.Z. Yue, Z.Z. Zhuo, et al., A stationary wavelet transform based approach to registration of planning CT and setup cone beam-CT images in radiotherapy. Journal of medical systems, 2014, 38: 40.

[19] R. Sharman, J.M. Tyler, O.S. Pianykh, A fast and accurate method to register medical images using wavelet Modulus Maxima. Pattern Recogn Lett, 2000, 21: 447-462.

[20] P. Angel, C. Morris, Analyzing the Mallat wavelet transform to delineate contour and textural features. Comput Vis Image Und, 2000, 80: 267-288.

Copyright $₫ 2015$ Shangli Cheng and Daxiang Cui. This is an open-access article distributed under the terms of the Creative Commons Attribution License, which permits unrestricted use, distribution, and reproduction in any medium, provided the original author and source are credited. 